\title{
e-Migrinter
}

$8 \mid 2012$

Regards sur les migrations sud-asiatiques

\section{Migrations au Maghreb}

Séminaire tenu lors de l'assemblée générale de Migrinter, à Poitiers, le 30 juin 2011

\section{Cédric Audebert}

\section{(2) OpenEdition}

Journals

Édition électronique

URL : https://journals.openedition.org/e-migrinter/648

DOI : $10.4000 /$ e-migrinter. 648

ISSN : 1961-9685

Éditeur

UMR 7301 - Migrinter

\section{Édition imprimée}

Date de publication : 4 avril 2012

Pagination : 119-123

ISSN : 1961-9685

\section{Référence électronique}

Cédric Audebert, « Migrations au Maghreb», e-Migrinter [En ligne], 8 | 2012, mis en ligne le 11 janvier 2016, consulté le 20 mai 2021. URL : http://journals.openedition.org/e-migrinter/648 ; DOI : https:// doi.org/10.4000/e-migrinter.648 
 \\ « Migrations au Maghreb », Séminaire tenu lors de 1'assemblée générale de Migrinter, Maison des Sciences de 1'Homme et de la Société de Poitiers, le 30 juin 2011
}

\author{
Compte rendu par Cédic Audebert
}

Note de la rédaction - Ce séminaire s'est déroulé fin juin 2011. Certains événements ont depuis largement évolués (fin du conflit entrainant d'autres mouvements, etc.). Publier ce compte rendu en l'état nous permet néanmoins de développer un regard rétrospectif sur les mouvements migratoires liés aux bouleversements de la région intervenus il y a buit mois.

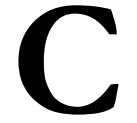

e séminaire avait pour objectif de faire le point sur les conséquences migratoires des mutations économiques et politiques, ainsi que sur les enjeux géopolitiques qui y sont liés. Il est vrai que les ressorts susceptibles d'influer sur la dynamique des migrations sont nombreux : la problématique de l'intégration à laquelle sont confrontées les générations issues de l'immigration maghrébine dans les pays européens, la crise économique des années 2000 , les bouleversements géopolitiques récents dans les pays du Maghreb ainsi que leurs mutations géoéconomiques internes conférant un nouveau rôle à des villes-interfaces comme par exemple Tanger.
Plus de quatre mois après le début de la crise libyenne, un million de réfugiés avaient quitté le pays : la moitié étant en Tunisie, 300000 en Égypte et 70000 au Niger. Le potentiel de déstabilisation politique des pays d'accueil, l'instrumentalisation de ces flux par le régime Kadhafi et les conditions de vie de plus en plus problématiques dans les camps donnent une nouvelle dimension à la question migratoire en Méditerranée.

Hassan Boubakri : Migrations, exil et révolutions au Sud de la Méditerranée

Entre fin février et fin juin 2011, entre 350000 et 500000 Libyens auraient fui vers la Tunisie, dont la moitié en provenance de la région de Tripoli, auxquels se sont ajoutés les réfugiés non libyens, venant surtout d'Egypte, d'Afrique subsaharienne (Somalie, Soudan, Erythrée, Côte d'Ivoire) et dans une moindre mesure d'Asie (en particulier du Bangladesh).

Les familles libyennes réfugiées peuvent être divisées en deux groupes : les pro-Kadhafi rentrés en Tunisie pour mettre leur famille à l'abri ; les originaires de régions rebelles (Misrata, etc.) passés par le sud. Un cinquième des familles libyennes a été accueilli dans les familles tunisiennes, avec des hommes jeunes repartant fréquemment au combat de l'autre côté de la frontière et transmettant de l'aide à partir de la Tunisie. Dans d'autres cas, de nombreux immigrés tunisiens en Europe ont fait acte 
de solidarité en appelant leur famille au pays pour que soit mise à disposition leur maison en Tunisie pour les réfugiés libyens. Beaucoup de familles libyennes n'ont pas souhaité s'installer dans les camps, ce qui explique la présence moins importante des ONG dans le sud du pays par rapport au nord. L'action des comités de protection de la révolution dans l'accueil des réfugiés y a donc été plus active.

Ces bouleversements politiques sont susceptibles d'avoir un impact durable sur les mutations migratoires dans la région et de produire dans les pays du Maghreb, en particulier en Tunisie, un nouveau discours du Sud vis-à-vis de l'Union européenne sur la question migratoire. Tout d'abord, l'explosion de l'immigration illégale tunisienne apparaît comme une continuité des mouvements migratoires antérieurs. Mais avec l'éclatement du conflit libyen se pose la question d'une remise en question des rapports antérieurs entre l'Union européenne et la Tunisie sur la question migratoire et notamment du contrôle par la Tunisie des flux migratoires subsahariens vers le Nord pour le compte de l'Union européenne.

Ensuite, malgré le départ massif de migrants de Lybie, il est probable qu'il y ait un retour massif de migrants (subsahariens et autres) en Lybie, pour la reconstruction du pays mais aussi parce que l'essentiel des corps de métiers traditionnels ont jusqu'ici largement reposé sur l'immigration. Concernant la circulation existante entre la Lybie et la Tunisie, un retour à la normale est probable, même si l'épineuse question des mercenaires (qui ne constituent que seulement $2 \%$ des Subsahariens en Lybie) risque de peser sur l'accueil futur des Subsahariens dans le pays.

\section{Zoubir Chatou : Mobilités transnationales et recompositions territoriales dans l'espace frontalier de Tanger}

Dans l'extrême nord du Maroc, Tanger (1,4 million d'habitants) connait une évolution singulière par rapport au reste $d u$ pays, avec une croissance démographique continue $(1,6 \%$ l'an $)$ et un taux d'urbanisation régional très supérieur à la moyenne nationale $(79 \%$ contre $56 \%$ ). Sur les quatre dernières années, la ville a gagné 120000 habitants, mettant à profit plusieurs atouts la rendant attractive pour les flux de migrations internes, avec pour conséquence un étalement de la ville sur les zones rurales avoisinantes annexées, lesquelles subissent alors une importante pression foncière. Sa situation géostratégique aux portes de l'Europe polarise un important trafic de produits licites et illicites (contrebande, cannabis, etc.), mais aussi le lancement de grands projets d'infrastructures: Port Tanger-Med, autoroutes, lignes ferroviaires, et implantation de grandes entreprises comme Renault. La ville a donc émergé comme un port transnational visant à concurrencer Algésiras ou Marseille.

Outre la visée économique, l'objectif est aussi politique car il s'agit de faire de Tanger une vitrine tant pour l'intérieur que l'extérieur du pays - et réduire au passage l'attractivité de l'enclave de Ceuta tout en renforçant la présence de l'Etat du Nord du Maroc («Tanger a tourné le dos au Maroc »). Mais force est de constater que les projets sont pensés et financés en dehors de la participation de la population locale, qui pourrait à terme être tentée de rejeter les nouveaux arrivants du reste du Maroc.

Enfin, la région est dynamisée par un autre phénomène: celui de l'installation de nouveaux entrepreneurs, les Marocains vivant à l'étranger (MRE). Ceux-ci créent de nouveaux espaces dans la ville où ils participent à un phénomène de gentrification. Souvent partis pour étudier, surdiplômés confrontés au chômage ou au sous-emploi en Europe, ces jeunes adultes reviennent en tant qu'entrepreneurs au pays. Ce défi constitue pour eux une manière de 
s'affranchir de l'image négative et de l'expérience mitigée vécue à l'extérieur du pays. Pour autant, ils sont confrontés dès leur retour à d'autres réalités économiques et sociales (bakchich, etc.) avec lesquelles ils doivent composer.

Tous ces changements introduisant de nouveaux acteurs liés aux migrations tant internes qu'externes sont porteurs d'espoirs mais aussi d'inquiétudes pour les populations locales dépassées par le phénomène.

\section{Mohamed Charef : Réflexions sur l'évolution de la recherche sur l'immigration au Maroc}

En trente ans, la réflexion scientifique sur les migrations marocaines a considérablement évolué, avec une prise en charge croissante de ces problématiques par les Marocains eux-mêmes, qu'ils soient à l'extérieur ou à l'intérieur du pays. À l'origine (années 1980), la question a été initiée dans les principaux pays d'immigration marocaine (France, Belgique) par les chercheurs de la seconde génération. Puis, le thème des actions de développement portées par les immigrés vis-à-vis des régions d'origine a rapidement émergé.

Si dans le contexte colonial puis postcolonial, la présence européenne a longtemps été mise en exergue dans les recherches migratoires locales (à travers les figures du colon, puis du coopérant et de l'expatrié), celle de l'émigré marocain réinvestissant socialement et affectivement le pays d'origine a progressivement émergé, dans un contexte où ses droits sont de plus en plus reconnus au Maroc.

La recherche s'est notamment de plus en plus intéressée à la question du lien entre migration et développement en mettant en relief l'implication des immigrés (en milieu urbain comme en milieu rural) et la nouvelle régionalisation qui en découle. Les émigrés jouent un rôle de plus en plus actif dans la décentralisation. Par exemple, les contraintes de développement auxquelles a été confronté le Souss (pénurie d'eau, désertification, enclavement, exode rural, pollution, etc.) ont constitué un défi auquel ont répondu les originaires de cette région vivant à l'étranger (Région parisienne, Nord de la France, etc.). Dans cette région marocaine où la tradition du travail solidaire communautaire restait bien ancrée, ils ont progressivement imposé l'association comme cadre d'action pour le développement local, au point de fréquemment se substituer à l'Etat dans la réalisation de projets structurants (réseau d'eau à usage domestique, électrification dans les communautés villageoises, routes, dispensaires, éducation préscolaire, alphabétisation des femmes rurales, transport scolaire, etc.). Des activités ont été créées pour stabiliser la population sur place, y compris par la réhabilitation des activités traditionnelles (poterie, gites ruraux, etc.).

Cette implication des émigrés ainsi que l'ouverture de l'Etat à la société civile et la coopération internationale qui y est en partie liée suscitent un certain nombre de questionnements et de pistes de recherches pour le futur. Les associations ont-elles une vision claire du développement sur le long terme ? Pour quel impact réel sur les régions concernées ? Les collectivités locales jouentelles le jeu dans l'esprit d'un partenariat complémentaire? Qu'en est-il de l'implication des universités et des médias dans ce processus? Quelles actions de grande envergure sont menées pour fédérer les efforts des associations à l'échelle des régions d'origine?

Juan David Sempere : Notes sur l'évolution des flux migratoires en Algérie

Dans un contexte de crise économique, la situation des immigrés s'est considérablement détériorée en Espagne et a eu des incidences sur la dynamique des flux migratoires dans l'ouest du bassin 
méditerranéen. En quelques années, ce pays, l'un des plus attractifs d'Europe pour les immigrés du Sud dans les années 1990 et 2000, est devenu un pôle migratoire plutôt répulsif sur le plan économique. Non seulement les immigrés ont modifié leurs projets de regroupement familial, ayant pour effet de renforcer la tendance au ralentissement de l'immigration, mais les Espagnols eux-mêmes ont commencé à émigrer.

Les investisseurs locaux regardent donc de plus en plus de l'autre côté de la Méditerranée, vers l'Algérie qui devient pour certains un nouvel eldorado. Il est vrai que dans l'optique d'endiguer la propagation de la contestation politique et de doper le niveau de vie, le gouvernement algérien a investi des milliards de pétrodollars dans divers secteurs de la vie économique. Faisant le parallèle avec la communication d'Hassan Boubakri, Juan David Sempere constate que cette conjoncture particulière est paradoxalement de nature à limiter les départs de migrants clandestins vers l'Europe, à la différence de ce qui a été observé pour le contexte tunisien. Parallèlement, la tendance à la diversification géographique de l'émigration d'universitaires algériens (vers le Canada ou le Golfe, par exemple) se poursuit.

Il semble donc que la crise politique au Sud de la Méditerranée n'ait pas eu les mêmes effets migratoires selon le pays concerné. Par exemple, Oran, qui attire les investisseurs espagnols, est aussi un nouvel eldorado pour les retraités britanniques qui traditionnellement ont jusqu'ici plutôt tendu à s'installer au Maroc voisin. Parallèlement aux effets sur la dynamique des migrations externes, les incidences sur les migrations internes à l'Algérie sont également notables. Effet des investissements de la manne pétrolière, la forte croissance des villes pétrolières de l'intérieur (Hassi Messaoud) et des villes du Nord (Alger, Oran) polarise l'exode rural.

\section{Les projets du Centre Jacques Berque (CJB, Maroc) et les migrations internationales}

Baudoin Dupret conclut le séminaire en proposant des pistes de collaborations entre le CJB et Migrinter. Est notamment évoquée l'importance de la thématique des migrations internationales au sein de l'observatoire des espaces transfrontaliers du $\mathrm{CJB}$, avec pour terrains potentiels l'Algérie, le Maroc, la Mauritanie, etc.

\section{Discussion}

Kamel Doraï lance le débat en rappelant quelques points forts récurrents dans les présentations, notamment la multiplicité de la nature des migrations articulant logiques économiques, politiques, etc. où apparaissent des passerelles entre les catégories (travailleurs qui deviennent des réfugiés et vice versa...).

Gildas Simon prend alors la parole en s'interrogeant sur la part de ce qui relève de la dynamique conjoncturelle, de celle relevant de changements plus structurels, dans les mutations migratoires récentes à l'œuvre en Tunisie. Quelle est notamment la part des logiques conjoncturelles politiques (émigration du fait d'une affiliation au régime de Ben Ali) ou économiques (perte d'emploi liée à la crise) par rapport à des logiques inscrites dans le temps long (celles d'un champ migratoire ancien) ? La question est étendue à d'autres espaces que la Tunisie, concernant de vieux champs migratoires européens, au départ de l'Espagne, de l'Irlande, du Portugal, de la Grèce, qui aujourd'hui semblent réactivés.

Abderrazek Oueslati prolonge la discussion en s'interrogeant sur le rôle des émigrés dans la reconstruction tunisienne.

Hassan Boubakri répond à Gildas Simon en soulignant trois éléments: $1^{\circ}$ ) l'importance des transferts d'argent dans le 
cadre de réseaux familiaux transnationaux pour le financement du voyage des jeunes candidats à l'émigration, et l'implication des émigrés en Italie dans le trafic de migrants; $2^{\circ}$ ) le caractère essentiellement économique de l'émigration de ces jeunes sans emploi (chômage massif, emploi saisonnier précaire dans l'agriculture et le tourisme); $3^{\circ}$ ) le bénéfice tiré de la crise libyenne dans le Sud tunisien qui, grâce à l'inversion des flux transfrontaliers de marchandises, a évité une crise économique majeure.

En réponse à Abderrazek Oueslati, Hassan Boubakri constate que les émigrés tunisiens en France tendent à devenir conservateurs dès lors qu'ils acquièrent une situation stable. Ils se positionnent contre les mouvements de clandestins, en reproduisant parfois les stéréotypes véhiculés par l'extrême droite.

Kamel Doraï réoriente la discussion en interrogeant la manière dont les Etats (en l'occurrence ici l'Etat tunisien) captent les ressources des ONG répondant à la présence de migrants réfugiés, en vue de renouveler les investissements dans le domaine de la santé et des infrastructures comme il l'a observé en Syrie.

Mohammed Charef intervient ensuite en soulignant l'implication économique et politique de la communauté marocaine à l'étranger qui, contrairement aux Marocains du Maroc, se sont montrés déçus des réformes proposées par le Roi. Dans un autre ordre d'idée, il rappelle que le Mali et le Nord du Tchad ont enregistré de nombreux retours de migrants dans des conditions difficiles suite à la crise en Libye.

Chadia Arab aborde la question de la représentativité des Marocains résidant à l'étranger dans la vie politique du pays d'origine, en soulignant que le CCME
(Conseil de la Communauté Marocaine résidant à l'Etranger) est élu par le parlement.

Hassan Boubakri pose ensuite la question du regard de l'Union européenne sur cette question migratoire, et Emmanuel Ma Mung celle d'une éventuelle institution représentative des Tunisiens à l'étranger. Hassan Boubakri répond alors que l'on s'oriente actuellement vers la création d'un Ministère des Tunisiens à l'étranger, et qu'il est prévu qu'une douzaine de députés les représentent au Parlement.

A la question de Gildas Simon sur les réfugiés qui restent "sur le carreau » dans le Sud tunisien - essentiellement les Erythréens, les Somaliens, les Bangladais Hassan Boubakri estime leur présence à au moins 3000 dans les camps du Sud actuellement où ils vivent dans des conditions difficiles.

En guise de conclusion, MarieAntoinette Hily rappelle que la problématique des migrations mobilise d'autres problématiques, comme celle de l'Etat comme forme culturelle (l'est-il encore?) ou du lien entre les formes de libéralisme économique et social et les aspirations de la population à des changements juridiques dans les pays du Maghreb (comme par exemple la question de la nouvelle Constitution au Maroc).

Cédric Audebert Géographe Chargé de recherche CNRS/ MIGRINTER - UMR 7301 cedric.audebert@univ-poitiers.fr 